\title{
Pulmonale Erkrankungen bei neurologischen Patienten
}

\author{
Peter Caspar Schulte
}

In einer allgemeinmedizinischen Praxis liegt der Anteil der Patienten mit pneumologischen Erkrankungen bei ca. 20 \%. Mit etwa der gleichen Häufigkeit erscheinen in der neurologischen Behandlung Patienten, die begleitend $\mathrm{zu}$ der neurologischen Erkrankung eine pneumologische Problematik aufweisen oder deren neurologische Erkrankung sich primär durch pulmonale Symptome bemerkbar gemacht hat. Des Weiteren gibt es eine Reihe neurologischer Erkrankungen, die im Verlauf zu bronchopulmonalen Störungen führen. Erkrankungen wie Tuberkulose, Vaskulitiden, Kollagenosen, aber auch Sarkoidose weisen sowohl neurologische als auch pneumologische Symptome auf mit gleichzeitig oder auch nacheinander auftretenden Beschwerden. In Anbetracht dieser doch oft sehr engen Verzahnung von Symptomatik und Klinik neurologischer und pneumologischer Erkrankungen erfolgt hier eine zusammenfassende Darstellung der für die Klinik wichtigsten pneumologischen Erkrankungen mit Hilfestellungen für die praktische klinische Arbeit. Dies gilt insbesondere für die medizintechnischen Entwicklungen der letzten Jahre wie die nCPAP-Therapie beim obstruktiven Schlafapnoe-Syndrom und alle Formen der nichtinvasiven und invasiven Beatmung bei durch neuromuskuläre Erkrankungen entstehenden Situationen. Diese erfordern eine enge Zusammenarbeit des Neurologen mit einem pneumologischen Kollegen mit Beatmungserfahrung in einem entsprechenden Zentrum.

\section{Asthma bronchiale}

Asthma bronchiale ist eine chronische Erkrankung der Atemwege, die mit einer Entzündung, einer reversiblen bronchialen Obstruktion und/oder mit einem hyperreagiblen Bronchialsystem einhergeht. Das klinische Bild ist sehr variabel und reicht von leichten Verläufen mit nur sehr geringer Symptomatik wie intermittierendem Husten oder Räusperzwang bis

P. C. Schulte $(\bowtie)$

Klinik für Pneumologie, Gastroenterologie und Innere Medizin, Alfried Krupp Krankenhaus, Essen, Deutschland

E-Mail: peter.schulte@krupp-krankenhaus.de hin zu intermittierend (ggf. anfallsartig) auftretender Luftnot mit subjektiv vom Patient empfundenem Pfeifen über der Lunge sowie auskultatorischem Giemen und Brummen bei der klinischen Untersuchung. Die Erkrankung beginnt typischerweise in der Kindheit, anamnestisch sind oft häufige Atemwegserkrankungen und Beschwerden in der frühen Kindheit und Jugend eruierbar. Diagnostisch wegweisend sind die Anamnese sowie die Befunde der Lungenfunktionsmessung.

Asthma ist eine der weltweit häufigsten Erkrankungen mit einer Prävalenz von ca. $10 \%$ aller Kinder, ca. $5 \%$ aller Erwachsenen; etwa 300 Millionen Menschen weltweit sind betroffen. Bezüglich der Mortalität sind weltweit etwa 250.000 Todesfälle pro Jahr infolge eines Asthma bronchiale zu vermuten (Buhl et al. 2017).

\section{Klassifikation}

In aller Regel ist das Asthma bronchiale veranlagungsbedingt, wir unterscheiden jedoch unterschiedliche Unterformen des Asthmas, die sich vor allen Dingen durch die Auslöser sowie den Phänotyp unterscheiden. Die Genese des Asthma bronchiale ist multifaktoriell. Die therapeutisch wichtigste Unterform ist das allergische Asthma bronchiale, da hier neben der rein symptomatischen Therapie auch eine kausale Therapie mittels Allergenkarenz sowie einer sogenannten spezifischen Immuntherapie (vormals Hyposensibilisierung) besteht. Zudem besteht seit einigen Jahren die Möglichkeit, dass allergische, vor allen Dingen IgEvermittelte Asthma sowie das eosinophile Asthma mittels sog. Biologicals zielgerichtet zu behandeln.

\section{Unterformen Allergisches Asthma bronchiale}

Hier lässt sich in aller Regel eine Sensibilisierung gegen ein oder mehrere Allergene mit Nachweis spezifischer IgEAntikörper (atopische Diathese) plus Allergenbezug zur Asthmasymptomatik nachweisen. Diese sehr häufige Unterform des Asthma bronchiale findet sich bei ca. $30 \%$ aller Asthmatiker und stellt gleichzeitig die häufigste Form des 
Asthma bronchiale bei Kindern dar. Auslösende Allergene können saisonal z. B. Pollen oder Pilzsporen sein, nichtsaisonal (perennial $=$ ganzjährig) findet sich häufig eine Sensibilisierung gegen Hausstaubmilben oder Tierepithelien. Besonderheiten der Therapie sind, dass durch eine Allergenkarenz wie z. B. Hausstaubmilbensanierung, Expositionsvermeidung bei Tierepithelallergie durch Abschaffen des Haustieres oder die Möglichkeit der spezifischen Immuntherapie eine kausale Therapieoption besteht.

\section{Nichtallergisches Asthma bronchiale}

Bei dieser Unterform findet sich im Gegensatz zum allergischen bzw. häufig auch gemischtförmigen Asthma bronchiale kein Nachweis spezifischer IgE-Antikörper und eine fehlende Allergiesymptomatik, diese häufigste Asthma-Unterform des Erwachsenen weist eine Prävalenz von ca. 30$50 \%$ aller erwachsenen Asthmatiker auf. Als auslösende Faktoren werden hier insbesondere Atemwegsinfekte, aber auch körperliche Anstrengung oder psychischer Stress diskutiert.

Mischform aus extrinsischem und intrinsischem Asthma bronchiale

Bei dieser Unterform finden sich sowohl eine Sensibilisierung gegen Allergene als Auslöser als auch die Besonderheiten des intrinsischen Phänotyps des Asthma bronchiale.

Einteilung nach Grad der Asthmakontrolle Nach Möglichkeit sollte bei allen Patienten ein kontrolliertes Asthma angestrebt werden (siehe Abschn. „Therapie“ und Tab. 1 und 2).

Einteilung nach Schweregrad Das Asthma bronchiale kann auch nach Schweregrad eingeteilt werden, dieser wird jedoch erst im Verlauf beim bereits therapierten Patienten festgelegt und nicht bei der Erstdiagnose. Er richtet sich nach der Asthmakontrolle sowie der Therapiestufe, auf welcher der Patient behandelt wird (siehe Abschn. „Therapie“ und Übersicht „Medikamentöse Langzeittherapie des Asthma bronchiale").

Leichtes Asthma Hierunter verstehen wir ein kontrolliertes Asthma bereits auf Therapiestufe 1 oder 2 .

Mittelgradiges Asthma Für eine Kontrolle des Asthmas ist eine Eskalation auf Therapiestufe 3 oder 4 notwendig.

Schweres Asthma Als schweres Asthma bezeichnen wir, wenn trotz Therapie mit inhalativen Kortikosteroiden (ICS) in Höchstdosis und mindestens einem zusätzlichen Langzeitmedikament (langwirksamen $\beta 2$-Mimetika - LABA oder Leukotrienrezeptor-Antagonisten - LTRA) oder oralem
Kortikosteroid für mehr als 6 Monate/Jahr noch einer der folgenden Punkte zutrifft:

- teilweise kontrolliertes oder unkontrolliertes Asthma

- mindestens eine Exazerbation mit stationärer Behandlung oder Beatmung in den letzten 12 Monaten

- mehr als zwei Exazerbation in den letzten 12 Monaten, die eine orale Steroidtherapie notwendig machten

- dauerhaft eingeschränkte Lungenfunktion, d. h. Einsekundenkapazität (FEV1) kleiner $80 \%$ des Sollwertes [FEV1/ forcierte Vitalkapazität (FVC) kleiner „lower limit of normal“"(LLN)]

\section{Klinik}

\section{Allgemeine Symptome}

Das Asthma äußert sich in aller Regel über einen chronischen Husten oder Räusperzwang, z. T. können diese auch das einzige klinisch fassbare Symptom bleiben. Des Weiteren bestehen eine teils anfallsartige, teils aber auch anhaltende Kurzatmigkeit und Dyspnoe. Häufig bestehen allergische Komorbiditäten wie eine chronische Rhinitis oder ein atopisches Ekzem.

\section{Exazerbation/Asthmaanfall}

Die Exazerbation bzw. der akute Asthmaanfall ist gekennzeichnet durch eine plötzlich auftretende Luftnot und exspiratorische Atemgeräusche mit Giemen, Brummen und pfeifender Atmung. Begleitend werden häufig eine Brustenge, Erstickungsangst sowie trockener Husten angegeben.

\section{Diagnostik}

\section{Diagnosekriterien des Asthma bronchiale}

Die Diagnose eines Asthma bronchiale erfordert neben der 3typischen Klinik und Anamnese den Nachweis einer in aller Regel reversiblen Bronchialobstruktion [FEV1/FVC (Tiffeneau-Index) $<\mathrm{LLN}]$ in der Lungenfunktion (durch Bronchospasmolysetest oder antiasthmatische Stufentherapie). Die Diagnose Asthma bronchiale gilt als gesichert bei charakteristischer Symptomatik und dem Nachweis einer (teil-)reversiblen Obstruktion! (Buhl et al. 2017).

Die Obstruktion, d. h. Atemflusslimitierung, resultiert aus mehreren Mechanismen. Durch eine Verkrampfung der glatten Muskulatur in der Bronchialwand (Bronchospasmus), ein Schleimhautödem durch eine entzündliche Infiltration durch verschiedene immunkompetente Zellen sowie deren Mediatoren sowie die Verlegung des Bronchiallumens durch Sekretion eines zähen Schleims (Dyskrinie) kommt es zu einer reversiblen Verengung der Atemwege. Langfristig kommt es zu einer Hyperplasie der Becherzellen und Verdickung der Basalmembran der Bronchien, im Rahmen des sog. Remodelings der Bronchialwände kommt es zudem zu 
Tab. 1 Grad der Asthmakontrolle (nach Buhl et al. 2017; Global Initiative for Asthma 2017)

\begin{tabular}{|c|c|c|c|}
\hline & $\begin{array}{l}\text { Kontrolliertes } \\
\text { Asthma }\end{array}$ & $\begin{array}{l}\text { Teilweise kontrolliertes Asthma (1-2 } \\
\text { Kriterien) }\end{array}$ & $\begin{array}{l}\text { Unkontrolliertes Asthma ( }>3 \\
\text { Kriterien) }\end{array}$ \\
\hline Symptome tagsüber & $<2$-mal/Woche & $>2-\mathrm{mal} /$ Woche & $>2-\mathrm{mal} /$ Woche \\
\hline Einschränkung von Alltagsaktivitäten & Nein & Ja & $\mathrm{Ja}$ \\
\hline Nächtliche Symptome oder nächtliches Erwachen & Nein & $\mathrm{Ja}$ & $\mathrm{Ja}$ \\
\hline $\begin{array}{l}\text { Notwendigkeit von Bedarfsmedikation oder } \\
\text { Notfallbehandlung }\end{array}$ & $<2$-mal/Woche & $>2-\mathrm{mal} /$ Woche & $>2$-mal/Woche \\
\hline $\begin{array}{l}\text { Lungenfunktion } \\
\text { FEV1, PEF (persönlicher Bestwert) }\end{array}$ & Normal & $<80 \%$ & $<80 \%$ \\
\hline Exazerbation & Nein & $<1-\mathrm{mal} / \mathrm{Jahr}$ & In dieser Woche \\
\hline
\end{tabular}

Tab. 2 Therapie der akuten Asthmaexazerbation

\begin{tabular}{|c|c|c|c|}
\hline Ablauf & Leichte Exazerbation & $\begin{array}{l}\text { Schwere } \\
\text { Exazerbation }\end{array}$ & Akuter Asthmaanfall \\
\hline $\begin{array}{l}\text { Klinische } \\
\text { Symptomatik }\end{array}$ & $\begin{array}{l}\text { Normales Sprechen } \\
\text { möglich, Atemfrequenz } \\
\text { normal }\end{array}$ & $\begin{array}{l}\text { Sprechdyspnoe, } \\
\mathrm{AF} \geq 25 / \mathrm{min}\end{array}$ & $\begin{array}{l}\text { Fehlendes Atemgeräusch (,Silent Lung“), Zyanose, Bradykardie/ } \\
\text { Hypotension bis hin zum Schock, } \mathrm{SaO}_{2}<92 \%, \mathrm{PaCO}_{2}>45 \mathrm{mmHg} \text { bei } \\
\text { Erschöpfung der Atemmuskulatur }\end{array}$ \\
\hline \multirow[t]{2}{*}{ Basistherapie } & \multicolumn{3}{|c|}{$\begin{array}{l}\text { Allgemeine Maßnahmen (atemerleichternde Manöver, z. B. Lippenbremse und atemerleichternde Körperposition, inhalative } \\
\text { kurzwirksame } \beta_{2} \text {-Sympathomimetika, z. B. Salbutamol) }\end{array}$} \\
\hline & Orale Glukokortikoide & \multicolumn{2}{|c|}{$\begin{array}{l}\text { Glukokortikoide p.o. oder i. v. (bei Kindern auch rektale Gabe möglich), Sauerstoff, ggf. } \\
\text { Ipratropiumbromid inhalativ }\end{array}$} \\
\hline $\begin{array}{l}\text { Erweiterte } \\
\text { Therapie }\end{array}$ & & & $\begin{array}{l}\beta 2 \text {-Sympathomimetika parenteral, Terbutalin s.c. oder Reproterol i. v., je } \\
\text { nach Klinik Intubation und invasive Beatmung }\end{array}$ \\
\hline \multirow[t]{2}{*}{ Versorgung } & Ambulante Versorgung & $\begin{array}{l}\text { Stationäre } \\
\text { Aufnahme }\end{array}$ & \multirow[t]{2}{*}{ Sofortige Verlegung auf die Intensivstation } \\
\hline & $\begin{array}{l}\text { Bei unzureichendem The } \\
\text { innerhalb von } 30-60 \mathrm{Mir} \\
\text { intensivmedizinische Ver }\end{array}$ & $\begin{array}{l}\text { ansprechen } \\
\text { ng erwägen }\end{array}$ & \\
\hline
\end{tabular}

einer Hypertrophie der glatten Muskulatur, sodass langfristig eine immer langsamer bzw. final nicht mehr reversible Obstruktion resultiert.

\section{Diagnostik bei akuter Asthmaexazerbation}

Neben einer gezielten Anamnese hinsichtlich möglicher Auslöser der akuten Symptomatik sollte eine eingehende klinische Untersuchung mit Auskultation sowie Inspektion des Thorax durchgeführt werden. Je nach klinischer Symptomatik sollten eine Pulsoxymetrie bzw. Blutgasanalyse sowie bei Hinweisen für einen Infekt auch ein Röntgenbild des Thorax zum Ausschluss bzw. Nachweis eines Infiltrates erfolgen. Je nach Schwere der Exazerbation sollten auch obligat im Labor Blutbild, Kreatinin, Elektrolyte sowie CRP bestimmt werden. In Abhängigkeit der Befunde muss dann eine Einschätzung des Schweregrades der Exazerbation sowie Einleitung der weiteren Therapie erfolgen.

\section{Therapie}

Bei der medikamentösen Therapie des Asthma bronchiale unterscheiden wir zwischen einer Dauertherapie (,Controller"s), die die Entzündung und bronchiale Engstellung dauerhaft bessern soll und einer Akuttherapie (,Reliever“), die im Falle einer raschen Aggravation der Beschwerden zu einer kurzfristigen Bronchialerweiterung führen soll. Als Controller kommen i. d. R. inhalative Kortikosteroide (ICS) und ggf. langwirksame bronchienerweiternden Medikamente, sog. langwirksame $\beta 2$-Mimetika zum Einsatz. Bei akuten Beschwerden kommen als Reliever schnellwirksame $\beta 2$ Sympathikomimetika (insbesondere Salbutamol) zum Einsatz, wenn notwendig, in Kombination mit einem systemischen Kortikosteroid. Um eine optimale Wirksamkeit der inhalativen Therapie zu gewährleisten, ist eine gute Patientenschulung essenziell, insbesondere zur korrekten und effektiven Inhalation in der Notfallsituation. Das in den gängigen Leitlinien beschriebene Stufenschema zur Therapie des Asthma bronchiale sieht je nach Verlauf und Beschwerdesymptomatik eine Eskalation bzw. Deeskalation der Medikation vor. Der akute Asthmaanfall stellt einen akuten Notfall dar, der einen sofortigen Transport in die Klinik rechtfertigt.

Stufentherapie Ziel der Therapie des Asthmas ist eine Symptomfreiheit unter möglichst niedrig dosierter Therapie mit möglichst wenigen Medikamenten. Die Therapie des Asthmas ist daher nicht starr an einzelne Schweregrade gebunden, sondern ist volatil abhängig von der klinischen Symptomatik im Sinne eines „step up/step-down“, d. h., bei entsprechender klinischer Symptomatik muss intensiver therapiert werden, bei geringer Symptomatik kann die Therapie analog reduziert werden kann. Die Initialtherapie richtet sich nach dem Grad der Asthmakontrolle (bei unbehandelten Patienten), wobei bei teilweise kon- 
trolliertem Asthma der Therapiebeginn mit Therapiestufe 2, bei unkontrolliertem Asthma mit mindestens Therapiestufe 3 erfolgt (Global Initiative for Asthma 2019, 2017).

Grundsätzlich unterscheidet man zwei mögliche Vorgehensweisen. Bei der sog. Step-up-Therapie wählt man zu Therapiebeginn die wahrscheinlich niedrigste notwendige Therapiestufe und eskaliert die Therapie dann nach Bedarf. Im Gegensatz hierzu sieht die sog. Step-down-Therapie bei Therapiebeginn eine Stufe über der wahrscheinlich notwendigen Therapiestufe vor, hier wird bei Erreichen der Asthmakontrolle die Therapie wieder deeskaliert. Welche Vorgehensweise gewählt wird, hängt von der Erfahrung und Vorliebe des Behandlers ab (siehe Übersicht unten).

Die Therapie sollte fortlaufend pneumologisch/internistisch überprüft und ggf. angepasst werden. Bei unter der etablierten Therapie nicht- oder teilkontrolliertem Asthma sollte eine Therapieeskalation, bei guter Asthmakontrolle über $\geq 3$ Monate eine Therapiedeeskalation erfolgen, bei klinischer Verschlechterung sollte eine sofortige Reeskalation erwogen werden.

\section{Medikamentöse Langzeittherapie des Asthma}

bronchiale, Stufenschema (NVL 2018)

- Stufe 1: Bedarfsorientierte Therapie mit SABA, in den neuen Empfehlungen wird bereits in Stufe 1 ein ICS als Langzeittherapeutikum empfohlen. Akuttherapie bei Bedarf mit SABA

- Stufe 2: Dauertherapie mit ICS in niedriger Dosis plus Bedarfstherapie mit SABA, alternativ bei Kindern LTRA. Akuttherapie bei Bedarf mit SABA

- Stufe 3: Dauertherapie mit ICS in niedriger oder mittlerer Dosis plus ein weiteres Langzeittherapeutikum; Bedarfstherapie mit SABA oder ICS/ Formoterol $*$, alternativ ICS/LAMA $* *$, niedrige Dosis oder ICS niedrige Dosis plus LTRA. Akuttherapie bei Bedarf mit SABA oder ICS/ Formoterol*

- Stufe 4: Dauertherapie mit ICS mittlere bis hohe Dosis plus $\geq$ ein weiteres Langzeittherapeutikum; Bedarfstherapie mit SABA oder ICS/Formoterol*. Therapiealternative ICS/LABA $* * *$ : Mittlere bis hohe Dosis plus LTRA oder ICS: Mittlere bis hohe Dosis plus LAMA**. Akuttherapie mit LABA oder ICS/Formoterol*

- Stufe 5: Ergänzende Therapie zu Stufe 4 durch Behandlung mit einem Biologikum (Anti-IgE**** oder Anti-IL-5*****). Alternativ dauerhaft orale Kortikoide. Akuttherapie bei Bedarf mit SABA oder ICS/Formoterol.

Anmerkungen:
$*$ Die Bedarfstherapie mit ICS/Formoterol erfolgt im Rahmen des sog. SMART-Konzepts, mit Verwendung einer fixen ICS/LABA-Kombination sowohl zur Langzeit- als auch zur Bedarfstherapie. Im Vordergrund steht bei der Akuttherapie der schnelle Wirkungseintritt des Formoterol.

**Aus der Gruppe der LAMA ist Tiotropiumbromid bei Asthma bronchiale zugelassen (ab 6 Jahren).

$* * *$ Langwirksame $\beta_{2}$-Sympathomimetika sind keine Monotherapeutika und werden nur in Kombination mit inhalativen Glucocorticoiden eingesetz; wirken insbesondere gut zur Prophylaxe der nächtlichen Asthmasymptomatik.

$* * * *$ Anti-IgE-Antikörper werden bei allergischem Asthma (ab dem Alter von 6 Jahren) in Stufe 5 zusätzlich empfohlen.

$* * * * *$ Anti-IL-5-Antikörper werden bei schwergradigem eosinophilem Asthma (ab dem Alter von 18 Jahren) in Stufe 5 zusätzlich empfohlen.

\section{Abkürzungen:}

$S A B A$ : kurzwirksame $\beta 2$-Mimetika: Salbutamol, Fenoterol, Terbutalin. ICS: inhalative Kortikosteroide: Budesonid, Fluticason. LTRA: Leukotrienrezeptor-Antagonisten: Montelukast. $L A B A$ : langwirksame B2-Sympathomimetika: Formoterol, Salmeterol. LAMA: langwirksame Muskarinrezeptor-Antagonisten (Anticholinergika): insbesondere Tiotropiumbromid. Kombinationspräparate ICS/LABA: Budesonid/Formoterol, Beclometason/Formoterol, Fluticasonpropionat/ Formoterol, Fluticasonpropionat/Salmeterol, Fluticasonfuroat/Vilanterol.

Biologicals: Anti-IL-5-Antikörper: Mepolizumab und Reslizumab; Anti-IgE-Antikörper: Omalizumab.

Therapie der akuten Asthmaexazerbation Die Therapie bei Exazerbation ist in Tab. 2 zusammengefasst.

\section{Chronisch obstruktive Bronchitis und Emphysem}

Die chronisch-obstruktive Lungenerkrankung (COPD) ist eine chronisch progrediente Atemwegserkrankung, bei der es vorwiegend durch inhalative Schadstoffe, insbesondere Tabakrauch, zu einer Entzündung der Bronchialschleimhaut, insbesondere im Bereich der kleinen Atemwege kommt. Dauerhaft führt dies $\mathrm{zu}$ einer irreversiblen Verengung der Bronchien mit konsekutiver Überblähung der Alveolen durch ein durch die verengten Bronchiolen - d. h. durch die Ent- 
zündung der kleinsten Atemwege - bedingtes Airtrapping. Langfristig resultiert hieraus eine irreversible Überblähung der Lunge einhergehend mit einem Verlust der Gasaustauschfläche (Emphysem). Hierdurch kommt es langfristig zu einer Hypoxie bzw. bei Erschöpfung der Atempumpe durch Verminderung der Ventilation zu einer Hyperkapnie.

Der vermehrte Energiebedarf für die Atemarbeit durch die erschwerte Atmung mit erschöpfendem Einsatz der Atemhilfsmuskulatur führt im Endstadium zum körperlichen Verfall (Kachexie). Im Verlauf der COPD kann es durch die sich entwickelnde Hypoxämie zu neurologischer Symptomatik bis zur Bewusstlosigkeit kommen.

Der überwiegende Teil der Fälle bei COPD ist Folge des Tabakrauchens. Die Leitsymptome der COPD sind die sog. AHA-Symptome (Atemnot, Husten, Auswurf). Die Diagnose der COPD kann meistens schon durch die Anamnese (Tabakrauchen) sowie die klinische Symptomatik gestellt werden, eine Lungenfunktionsuntersuchung kann die Diagnose dann sichern und dient der Schweregradeinteilung der COPD. Anhand des Schweregrades kann dann unter Zusammenschau weiterer klinischer Parameter eine stadiengerechte symptomatische Therapie mit Bronchodilatatoren bzw. antiinflammatorischen Medikamenten eingeleitet werden. Die wirksamste Maßnahme zur Vorbeugung eines Progresses der Erkrankung ist jedoch die Ausschaltung der auslösenden Noxe, sprich strikte Nikotinkarenz.

Männer sind insgesamt deutlich häufiger betroffen als Frauen, weltweit rangiert die COPD als dritthäufigste Todesursache (WHO 2018), die Prävalenz ist mit zunehmendem Alter ansteigend, die höchste Prävalenz besteht bei Patienten $>70$ Jahre. Die Prävalenz für die fortgeschrittenen Stadien II-IV in Deutschland beträgt etwa $13 \%$.

\section{Klassifikation}

Die vormals übliche Einteilung der Schwere der COPD nach der Lungenfunktion wurde in den letzten Jahren verlassen und die Relevanz der Spirometrie (FEV1-Ergebnis) wird in den aktuellen Leitlinien heruntergestuft (Vogelmeier et al. 2018a, b). Anstelle eines starr an der Lungenfunktion ausgerichteten Therapieschemas steht nun eine individualisierte Therapie, welche sich an der klinischen Einteilung in Patientengruppen nach Gold orientiert. Eine kombinierte Einschätzung berücksichtigt weiterhin den Schweregrad der Obstruktion, etwaige Diskrepanzen zwischen objektiver Obstruktion und Symptomschwere werden damit einbezogen.

Einteilung in Patientengruppen nach GOLD Die Einteilung nach Gold bewertet vorrangig die Symptomschwere anhand von Fragebögen sowie die Anzahl der vorausgegangenen Exazerbationen (Vogelmeier et al. 2018b, Tab. 3), standardisierte Befragungsinstrumente sind die mMRCDyspnoe-Skala und der COPD Assessment Test (CAT).
Tab. 3 Patientengruppen nach Gold (Vogelmeier et al. 2018b)

\begin{tabular}{|l|l|l|}
\hline & Exazerbationen pro Jahr & Klinische Symptomatik \\
\hline Gold A & $<1$ & Wenig symptomatisch \\
& & mMRC $<2$ \\
& CAT $<10$ \\
Gold B & & $\begin{array}{l}\text { Stark symptomatisch } \\
\text { mMRC }>2\end{array}$ \\
& & CAT $>10$ \\
Gold C & $>2$ & Wenig symptomatisch \\
& & mMRC $<2$ \\
& & CAT $<10$ \\
Gold D & & Stark symptomatisch \\
& & mMRC $>2$ \\
& & CAT $>10$
\end{tabular}

mMRC-Dyspnoe-Skala (Modified Medical Research Council) Die Skala ist ein Instrument zur Graduierung der COPD auf Basis der Schwere der Dyspnoe (nach Belastungstoleranz) und des Einflusses auf die Alltagsaktivitäten (nach Gold Report 2011).

0 Atemnot nur bei starker körperlicher Belastung

1 Atemnot bei schnellem Gehen und leichtem Bergaufgehen

2 Vermeidungsverhalten, geht langsamer als Gleichaltrige ohne Erkrankung bzw. benötigt beim Gehen Pausen zur Erholung

3 Benötigt beim Gehen nach $100 \mathrm{~m}$ Strecke oder wenigen Minuten eine Pause zur Erholung

4 Verlässt das Haus nicht mehr und ist wegen Dyspnoe kaum noch in der Lage, sich selbstständig zu versorgen

Interpretation: Ein mMRC-Wert $\geq 2$ spricht für das Vorliegen einer schweren Symptomatik.

CAT (COPD Assessment Test) Siehe Fragebogen auf der Homepage der Deutschen Gesellschaft für Pneumologie (https://www.atemwegsliga.de/copd-assessment-test.html), das Dokument ist urheberrechtlich geschützt.

Interpretation:
0-10 Punkte:
11-20 Punkte:
Geringe individuelle Symptomatik
-20 Punkte: Mittelgradige individuelle Symptomatik
$\geq 20$ Punkte: Ausgeprägte individuelle Symptomatik

Einteilung nach Schweregrad der Obstruktion in der Spirometrie Basis dieser Einteilung ist die Lungenfunktionstestung mit Messung des Tiffeneau-Index und der FEV1 (Einsekundenkapazität) (Tab. 4).

\section{Klinik}

Leitsymptome der COPD sind chronischer Husten und Auswurf, typisch ist das morgendliche Abhusten von Sputum (Raucherhusten), ferner Dyspnoe, initial als Belastungsdyspnoe, im Verlauf jedoch als dauerhafte Dyspnoe (,AHA“ = Auswurf, Husten, Atemnot). Bei langjähriger Hypoxie können 
Tab. 4 Einteilung der COPD nach Schweregrad der Obstruktion in der Spirometrie (FEV1) (Vogelmeier et al. 2018a, b)

\begin{tabular}{|l|l|l|}
\hline $\begin{array}{l}\text { Schweregrad der } \\
\text { Obstruktion }\end{array}$ & $\begin{array}{l}\text { Tiffeneau-Index } \\
(\text { FEV1/VC) }\end{array}$ & $\begin{array}{l}\text { FEV1 \% vom } \\
\text { Soll }\end{array}$ \\
\hline COPD Grad I & $<70 \%$ & $>80 \%$ \\
\hline COPD Grad II & & $50-79 \%$ \\
\hline COPD Grad III & & $30-49 \%$ \\
\hline COPD Grad IV & & $<30 \%$ \\
\hline
\end{tabular}

Stigmata wie Uhrglasnägel und Trommelschlegelfinger sowie ein Fassthorax als Hinweis für ein Emphysem auftreten.

\section{Diagnostik}

Die primäre Diagnostik sollte eine eingehende Anamnese, insbesondere hinsichtlich der Rauchgewohnheiten, aber auch der beruflichen Belastungen, sowie eine eingehende körperliche Untersuchung umfassen, je nach Klinik sollten eine Blutuntersuchung und eine Pulsoxymetrie erfolgen, bei Sauerstoffsättigung $\left(\mathrm{SaO}_{2}\right)<94 \%$ oder jedem kleinsten Verdacht auf eine Hyperkapnie ist eine BGA indiziert.

Die apparative Diagnostik sollte obligat eine Lungenfunktionstestung mit Spirometrie, idealerweise jedoch mit Bodyplethysmografie und Messung der Diffusionskapazität umfassen, typische Befunde sind hier ein erniedrigter Tiffeneau-Index und erniedrigte FEV1, häufig in Kombination mit einem erhöhten Residualvolumen durch eine konsekutiv auftretende Überblähung. Ein Tiffeneau-Index $>70 \%$ schließt eine COPD weitgehend aus.

Mittels Bronchospasmolysetest kann eine Differenzierung zu einem Asthma bronchiale erfolgen, im Gegensatz zu diesem zeigt sich bei der COPD lediglich eine geringe bzw. fehlende Reversibilität der Obstruktion (kein wesentlicher Anstieg von FEV1 bei COPD, $\Delta$ FEV1 $<12 \%$ bzw. $<200 \mathrm{ml}$ ). Bei Verdacht auf eine Pneumonie sollte zudem abhängig von der klinischen Symptomatik ein konventionelles Röntgen-Thorax erfolgen. Aufgrund der Risikokonstellation der Patienten mit häufig langjähriger Nikotinanamnese kann zusätzlich bei entsprechendem Verdacht auch ein CT des Thorax zum Ausschluss bzw. Nachweis höherwertiger struktureller Veränderung der Lunge bzw. eines Malignoms diskutiert werden.

\section{Therapie}

\section{Allgemeine Therapieempfehlungen}

- Nikotinkarenz, Tabakentwöhnung

- Durchführung der empfohlenen Impfungen hinsichtlich respiratorischer (Influenzaviren jährlich, PneumokokkenImpfung)

- Patientenschulung

- Physikalische Maßnahmen

- Körperliche Aktivität im Rahmen ambulanter oder stationärer Rehabilitationsmaßnahmen, Lungensport erhält Be- lastbarkeit, lindert Dyspnoe u. a. durch Verbesserung der Hypoxämietoleranz

- Osteoporoseprophylaxe mit Vitamin D3 und Kalzium

Medikamentöse Therapie

Siehe folgende Übersicht.

Medikamentöse Therapie der COPD als Stufentherapie nach aktuellen Empfehlungen (Vogelmeier et al. 2018a, b)

- COPD Gruppe A nach Gold:

- Dauertherapie nicht unbedingt erforderlich, ggf. bedarfsweise SAMA und SABA (initial)

- In den neueren Empfehlungen sollte eine Monotherapie mit LAMA oder LABA erwogen werden, als Bedarfsmedikation sollten SABA und/ oder SAMA verwendet werden.

- COPD Gruppe B nach Gold:

- Monotherapie mit LAMA oder LABA, als Alternative bei nicht ausreichendem Ansprechen kann eine 2er-Kombination LAMA und LABA verabreicht werden.

- Als Bedarfsmedikation sollen SABA und/oder SAMA verwendet werden.

\section{- COPD Gruppe C nach Gold:}

- Monotherapie, vorzugsweise LAMA, bei nicht ausreichendem Ansprechen kann bei Initialtherapie mit LAMA zusätzlich ein LABA, idealerweise als Kombinationspräparat bzw. Kombination LABA/LAMA und ICS erwogen werden.

- Als Bedarfsmedikation sollen SABA und/oder SAMA verwendet werden.

\section{- COPD Gruppe D nach Gold:}

- Hier sollte obligat eine 2er-Kombination LAMA und LABA verwendet werden, bei asthmatischer Komponente und/oder Eosinophilie sollte aufgrund des häufig guten Ansprechens bereits primär eine 3er-Kombination LABA/LAMA/ + ICS verwendet werden.

- Bei fehlendem Ansprechen oder therapierefraktärer Symptomatik kann auch ohne Asthma oder Biomarker auf eine Triple-Therapie LABA/ LAMA/ + ICS gewechselt werden. Ebenso kann der Einsatz von Phosphodiesterase-4(PDE-4)Hemmer (Roflumilast) erwogen werden. Bei $\geq 2$ Exazerbation pro Jahr oder aber Nachweis von Pseudomonas aeruginosa kann auch eine Langzeit-Antibiose mit einem Makrolid versucht werden (keine endgültig eindeutige Empfehlung in den Leitlinien hierzu). 
Therapie von akuten Exazerbationen (AECOPD) Der Großteil der akuten Exazerbationen ist infektbedingt (v. a. in Winter und Herbst), häufige Erreger sind Haemophilus influenzae und Streptococcus pneumoniae oder Viren (Matthys und Seeger 2008).

Die Exazerbation ist gekennzeichnet durch zunehmende Atemnot mit respiratorischer Insuffizienz. Eine Exazerbation der COPD kann durchaus schnell aggravieren und zu einem lebensbedrohlichen Notfall werden. Daher ist eine gute initiale Einschätzung bei Erstkontakt erforderlich und je nach Ausprägung der Symptomatik muss ebenfalls eine Versorgung auf der Überwachungsstation/Intensivstation erfolgen.

Die Therapie variiert je nach klinischer Ausprägung. Neben einer sitzenden Lagerung sollte eine inhalative Therapie mit $\beta 2$-Sympathikomimetika (z. B. Salbutamol) sowie Parasympathikolytika (z. B. Ipratropiumbromid) eingeleitet werden. Beide Substanzen haben in Kombination einen guten synergistischen Effekt bei der Bronchodilatation und können inhalativ über einen Druckluftvernebler appliziert werden. Auf eine ausreichende Flüssigkeitsgabe unter Berücksichtigung kardialer Begleiterkrankungen sollte geachtet werden.

\section{Therapieempfehlungen}

- Glukokortikoide systemisch intravenös oder oral in der Akutsituation 5-7 Tage

- Morphin i. v. oder s.c. in individuell adaptierter Dosierung zur Beruhigung bei starker Dyspnoe

- Inhalative $\beta 2$-Sympathikomimetika und Parasympathikolytika (z. B. Salbutamol, Ipratropiumbromid 3bis 4-mal täglich über Druckluftvernebler): bei ungenügendem Ansprechen auf die inhalative Therapie z. B. Reproterol i. v. oder bei fehlendem Venenzugang Terbutalin s.c.

- Antibiotische Therapie der AECOPD (Bodman et al. 2018; Vogelmeier et al. 2018b): Indiziert bei purulentem Sputum und Hinweisen auf eine bakterielle Infektion: z. B. Aminopenicillin +/-Betalaktamase-Inhibitor (Bodman et al. 2018; Vogelmeier et al. 2018a). Bei fehlender Purulenz konservatives Vorgehen ohne antibiotische Therapie

- Sauerstoffgabe: Über Nasenbrille, aufgrund der drohenden schweren Hyperkapnie langfristig nicht mehr als 1-2 1/min, Zielwert: $\mathrm{SpO}_{2} 85-95 \%$ bei fortgeschrittener COPD, obligat BGA-Kontrolle 30-60 Minuten nach Einleitung der Sauerstofftherapie

- Beatmung: Bei zunehmender respiratorischer Insuffizienz: nichtinvasive Beatmung (NIV); invasive Beatmung als Ultima Ratio, engmaschige BGAKontrollen unter einer NIV. Bei Verschlechterung der Hyperkapnie und Azidose Intubationsindikation prüfen. NIV-Toleranz medikamentös unterstützen: Individuelle Dosistitration von Morphin zur Anxiolyse und oberflächlichen Sedierung, der Patient sollte jedoch ansprechbar bleiben

Die invasive Beatmungstherapie kann beim akut erkrankten Patienten mit terminaler COPD auch zu einer dauerhaften Abhängigkeit vom Beatmungsgerät führen - darüber sollten Patienten im Verlauf ihrer progredienten Erkrankung rechtzeitig ärztlich aufgeklärt werden!

\section{Pneumonie}

Unter einer Pneumonie verstehen wir eine Entzündung des Lungengewebes, d. h. des Alveolarkammes und/oder des dazwischen gelegenen Bindegewebes. Die Pneumonie stellt eines der größten Probleme im Gesundheitswesen dar und ist die am häufigsten zum Tode führende Infektionserkrankung in den westlichen Industrienationen. Die häufigsten Erreger bei der infektiösen Pneumonie sind Bakterien, mit einem je nach Altersgruppe und Infektionsursache (ambulant oder im Krankenhaus erworben) unterschiedlichen Spektrum. Die häufig durch Pneumokokken verursachte ,klassische“ Pneumonie geht mit einem plötzlich einsetzenden schweren Krankheitsgefühl, produktivem Husten und hohem Fieber einher. Bei der klinischen Untersuchung imponieren auskultatorisch feinblasige, sog. klingende Rasselgeräusche über dem betroffenen Lungenabschnitt. Korrespondierend zu den klinischen Infektzeichen finden sich laborchemisch erhöhte Entzündungswerte. In der bildgebenden Diagnostik der Lunge findet sich in aller Regel ein Lungeninfiltrat.

Der Verlauf und die Symptomatik der Pneumonie sind sehr variabel, so kann die Erkrankung insbesondere bei älteren oder immunsupprimierten Patienten oder bestimmten Erregern (z. B. Viren, Mykoplasmen) atypisch mit abgeschwächten Symptomen und ohne physikalische Zeichen einer Infiltration verlaufen. Das einzige obligate Kriterium für die Diagnose einer Pneumonie ist damit ein neu aufgetretenes Infiltrat im Röntgenbild der Lunge. Neben einer körperlichen Schonung sowie ausreichender Hydrierung des Patienten sollte bei einer bakteriellen Pneumonie eine erregergerechte antibiotische Therapie erfolgen.

\section{Ätiologie}

Bei der Therapie muss berücksichtigt werden, ob es sich um eine sog. Community-acquired Pneumonia (CAP), d. h. ambulant erworbene Pneumonie, Hospital-acquired Pneumonia (HAP), also im Krankenhaus erworbene Pneumonie, oder neuerdings auch Institution-acquired Pneumonia (IAP), d. h. in stationärer oder teilstationärer Einrichtung bzw. dau- 
erhafter häuslicher Pflegeeinrichtung erworbene Pneumonie, handelt. Dies ist vor allen Dingen wichtig für die Auswahl der Antibiotika bei einer kalkulierten Therapie, da jede Art der Pneumonie ein bestimmtes Erregerspektrum als häufigster Erreger umfasst.

Pneumonien zeichnen sich durch ein vielfältiges Erregerspektrum aus (Bakterien, Viren, seltener Pilze), wobei Faktoren wie das Alter, die Umstände der Infektion (ambulant vs. nosokomial), aber auch regionale und saisonale Faktoren, Begleiterkrankungen sowie vorausgegangene Antibiotikabehandlungen Einfluss auf das Erregerspektrum haben können.

- Ambulant erworbene Pneumonie: Pneumokokken, Haemophilus influenzae, Mycoplasma pneumoniae und Chlamydia pneumoniae

- Seltenere bakterielle bzw. virale Erreger einer Pneumonie im ambulanten Bereich sind Legionellen, Staphylococcus aureus, Enterobacteriaceae, Bordetella pertussis (Impfstatus), Mycobacterium tuberculosis, primäre Viruspneumonie durch Influenza, Respiratory-Syncytial-Virus (RSV), Adenovirus, Masern, Metapneumovirus, Hantavirus, SARS (Reiseanamnese)

- Nosokomial erworbene Pneumonie: v. a. gramnegative Erreger (wie Pseudomonas aeruginosa und Enterobacteriaceae), Staphylokokken

- Pneumonie bei Immunsuppression: Häufig ausgelöst durch opportunistische oder bei Immunkompetenten nicht sehr virulente Erreger, z. B. Pneumocystis jirovecii, Aspergillus fumigatus, Candida species, Zytomegalievirus

- Möglich sind auch fulminante Verläufe (z. B. beidseitige Pneumonien) durch Erreger des normalen Spektrums.

Risikofaktoren für die Entwicklung einer Pneumonie sind hohes Alter und Immobilität jeglicher Ursache (Schlaganfall!), ein schlechter Immunstatus, chronische Vorerkrankungen wie kardiopulmonale Erkrankungen, beispielsweise Asthma bronchiale, COPD, Herzinsuffizienz, aber auch erworbene oder angeborene Alteration der Atemwege wie Bronchiektasen, raumfordernde Prozesse wie Tumoren oder Mukoviszidose. Des Weiteren begünstigen chronische Infektion der Mundhöhle, residuelle Zustände nach Operation im HNO-Gebiet sowie Schluckstörungen die Entstehung von Pneumonien.

Die nosokomiale Pneumonie, die Aspirationspneumonie und die durch spezielle Erreger ausgelösten Pneumonien haben in der klinischen Präsentation und Diagnostik ihre Besonderheiten und benötigen spezielle Therapieregime.

\section{Diagnostik}

Klinische Untersuchung und Erfassung der erweiterten Vitalzeichen (Puls, Blutdruck, Atemfrequenz, Temperatur rektal, Sauerstoffsättigung).

\section{Apparative Diagnostik}

- Konventionelles Röntgen-Thorax

- CT-Thorax bei unklaren Befunden im Röntgen-Thorax zur Beurteilung von Verschattungen, Einschmelzungen oder Pleuraempyem

- Pleurasonografie, ggf. Pleurapunktion bei Vorliegen eines Pleuraergusses mit Bestimmung des $\mathrm{pH}$, Beimpfung von Blutkulturflaschen mit dem Punktat

- Bronchoskopie: bei schweren Pneumonien zum Erregernachweis und gezielter Antibiotikatherapie. Wegen der Gefahr der respiratorischen Insuffizienz bei ursprünglich spontan atmenden Patienten sorgfältig abwägen! Keine Evidenz für routinemäßigen Einsatz!

\section{Labordiagnostik}

- Blutbild, CRP, Procalcitonin (PCT; höchste Spezifität für den Nachweis einer bakteriellen Pneumonie), Blutgasanalyse (BGA), um respiratorische Insuffizienz auszuschließen

- Ergänzendes Basislabor mit Transaminasen, Quick/INR, Kreatinin, Harnstoff, Blutzucker

- Erregerdiagnostik bei stationärer Aufnahme, d. h. obligat Blutkulturen, mindestens jeweils zwei aerobe und anaerobe Kulturen, Urin auf Legionellenantigen, Sputumkultur, ggf. erweiterte mikrobiologische und serologische Erregerdiagnostik

Diagnostische Kriterien Die Diagnose einer Pneumonie kann gestellt werden bei gemeinsamem Auftreten von einem Haupt- und 2 Nebenkriterien.

- Hauptkriterium:

- Neu aufgetretenes Infiltrat im konventionellen Röntgen-Thorax in zwei Ebenen

- Nebenkriterien:

- Fieber $\left(\geq 38,5^{\circ} \mathrm{C}\right)$ oder Hypothermie $\left(\leq 36,5{ }^{\circ} \mathrm{C}\right)$

- Leukozytose $(>10.000 / \mu \mathrm{l})$ oder Leukopenie $(<4000 / \mu \mathrm{l})$

- Eitriger Auswurf (gelblich-grün)

- Auf Infiltration hinweisende physikalische Zeichen (Bronchophonie, Stimmfremitus), nur geringe Sensitivität und Spezifität

- Nachweis eines Erregers in Blutkultur, Sputum, Bronchialsekret oder Pleuraflüssigkeit (DGP et al. 2015)

Die Diagnose einer Pneumonie kann im klinischen Alltag durchaus Schwierigkeiten bereiten, da symptomarme Verläufe vor allen Dingen bei chronisch vorerkrankten Patienten, vorerkrankter Lunge oder aber begleitender Immunsuppression möglich sind.

\section{Therapie}

Die Therapie der Pneumonie erfolgt entsprechend des Schweregrads bzw. des individuellen Verlaufs. Sie ist multi- 
modal und beinhaltet sowohl symptomatisch-supportive nichtmedikamentöse und medikamentöse Maßnahmen als auch eine antibiotische Therapie. Wenn möglich, wird sie nach Erregernachweis von einer kalkulierten Initialtherapie auf eine spezifische Therapie umgestellt. Die Indikation für eine stationäre Aufnahme ist abhängig von Klinik, Alter und Risikofaktoren (CRB-65-Score, s. unten).

Stationäre Aufnahme CRB-65-Score (Bauer et al. 2006):

\begin{tabular}{|c|c|}
\hline $\mathrm{C}$ & „Confusion“ (Bewusstseinseintrübung) \\
\hline $\mathrm{R}$ & „Respiratory Rate“ (Atemfrequenz $\geq 30 / \mathrm{min}$ ) \\
\hline B & $\begin{array}{l}\text { „Blood pressure“ (diastolischer Blutdruck } \\
\leq 60 \mathrm{mmHg} \text { oder systolischer Blutdruck } \\
<90 \mathrm{mmHg} \text { ) }\end{array}$ \\
\hline 65 & „Age“ (Alter $\geq 65$ Jahre) \\
\hline $\begin{array}{l}\text { Punkte nach } \\
\text { CRB-65-Score }\end{array}$ & Interpretation \\
\hline$\leq 1$ & Ambulante Führung möglich \\
\hline$\geq 2$ & Stationäre Behandlung indiziert \\
\hline$\geq 3$ & $\begin{array}{l}\text { Erhöhtes Komplikationsrisiko, ggf. Aufnahme } \\
\text { auf die Intensivstation erwägen }\end{array}$ \\
\hline
\end{tabular}

Jeder ambulant behandelte Patient mit Pneumonie sollte nach 48-72 Stunden erneut untersucht werden, um die Wirksamkeit des verschriebenen Antibiotikums zu prüfen.

Symptomatisch-supportive Maßnahmen Supportive Maßnahmen umfassen körperliche Schonung, jedoch keine strenge Bettruhe. Frühmobilisierung und Atemtherapie zur Sekretmobilisation sollten von Beginn an erfolgen. Bei Hypoxie sollte eine Atemunterstützung bzw. Sauerstoffgabe, ggf. mittels High-flowDevice bzw. in Kombination mit einer nichtinvasiven Beatmung erfolgen.

Medikamentöse Therapie der ambulant erworbenen Pneumonie (DGP et al. 2015) Kalkulierte antibiotische Therapie bei Patienten mit leichter Pneumonie

Bei CRB-65 = 0 und ausreichender Oxygenierung $\left(\mathrm{SaO}_{2} \geq 90 \%\right)$ kann von einer leichten Pneumonie ausgegangen werden, die Behandlung erfolgt in aller Regel ambulant.

Kalkulierte antibiotische Therapie bei Patienten ohne Risikofaktoren

Aminopenicillin (z. B. Amoxicillin), bei PenicillinAllergie oder -Unverträglichkeit bzw. Verdacht auf Beteiligung atypischer Erreger Fluorchinolone der Gruppe III oder IV (Levofloxacin oder Moxifloxacin), Makrolid (z. B. Clarithromycin oder Roxithromycin), Doxycyclin.

Kalkulierte antibiotische Therapie bei Patienten mit Risikofaktoren/Komorbiditäten

Aminopenicillin + Betalaktamase-Inhibitor (z. B. Amoxicillin/Clavulansäure). Bei Verdacht auf Beteiligung atypi- scher Erreger Kombination mit einem Makrolid (z. B. Clarithromycin), bei Penicillinallergie oder Unverträglichkeit Fluorchinolone der Gruppe III oder IV (Levofloxacin oder Moxifloxacin).

Früher wurden sehr häufig gerade in der ambulanten Therapie orale Cephalosporine, z. B. Cefuroxim, eingesetzt. Diese sollten allerdings nicht mehr zur Anwendung kommen, da sie häufig nicht ausreichend wirksam sind und die Gefahr einer Selektion, z. B. von multiresistenten gramnegativen Bakterien (MRGN) bzw. Clostridium difficile, begünstigen. Ebenfalls sollten neuerdings die eigentlich gerade im ambulanten Bereich sehr gebräuchlichen Fluorchinolone Levofloxacin und Moxifloxacin nicht mehr angewendet werden, da beide Substanzen ungünstige Nebenwirkungsprofile im Sinne von Innenrupturen aufweisen. Sie sollten daher nur als Reservemittel angewandt werden. Nach persönlichen Erfahrungen des Autors haben jedoch beide Präparate, die ja jahrelang bewährt waren, weiterhin ihren Stellenwert in der Behandlung der Pneumonie und sollten in Fällen mit einer schlechteren Alternative gegeben werden.

Mittelschwere und schwere Pneumonie - in stationärer Behandlung (WHO 2018; DGP et al. 2015) Kalkulierte antibiotische Therapie bei Patienten mit mittelschwerer Pneumonie

Aminopenicillin plus Betalaktamase-Inhibitor (z. B. Ampicillin/Sulbactam) oder Cephalosporin der 2. oder 3. Generation (z. B. Cefuroxim oder Ceftriaxon), Kombination mit einem Makrolid zur Abdeckung atypischer Erreger und Nutzung immunmodulatorischer Effekte (z. B. Clarithromycin, Azithromycin).

Da Makrolide zu einer Verlängerung der QT-Zeit und damit Herzrhythmusstörung führen können, gibt es für deren Anwendung keine generelle Empfehlung. Bei ausbleibendem Effekt der Makrolide bzw. nach negativem LegionellaSchnelltest können die Makrolide nach 3 Tagen abgesetzt werden. Alternativ (z. B. bei Penicillin-Allergie) können die aktuell geschmähten Fluorchinolone der Gruppe III oder IV mit guter Pneumokokken-Wirksamkeit (z. B. Levofloxacin oder Moxifloxacin) zum Einsatz kommen, hier kann dann auf das Makrolid bei guter Wirksamkeit dieser Substanzen gegen atypische Erreger verzichtet werden.

Kalkulierte antibiotische Therapie bei Patienten mit schwerer Pneumonie

Die schwere Pneumonie geht einher mit akuter respiratorischer Insuffizienz und/oder septischem Schock und/oder dekompensierter Komorbidität (z. B. kardiale Dekompensation, exazerbierte COPD, akutes Nierenversagen) und sollte zwingend stationär, ggf. auch auf der Intensivstation behandelt werden. 
- Piperacillin/Tazobactam oder Cephalosporine der 3. Generation (z. B. Ceftriaxon), ggf. zusätzlich ein Makrolid (z. B. Clarithromycin)

- Alternative für Patienten ohne septischen Schock: Fluorchinolone der Gruppe III oder IV mit guter Pneumokokken-Wirksamkeit (z. B. Levofloxacin oder Moxifloxacin).

Bei Patienten mit schwerer ambulant erworbener Pneumonie sollte sofort eine Antibiotikatherapie eingeleitet werden!

Grundsätze der Antibiotikatherapie Über die Dauer der antibiotischen Therapie wurde in der Vergangenheit viel diskutiert. Als Faustregel gilt, dass eine antibiotische Therapie von 7 Tagen ausreichend erscheint. Generell kann die Medikation etwa 3 Tage nach Entfieberung abgesetzt werden, vorausgesetzt, das Ansprechen konnte mittels Bestimmung des Procalcitonins verkürzt werden (Therapieende bei PCT $\leq 0,25 \mu \mathrm{g} / \mathrm{l})$.

\section{- Unkritische Antibiotikaverordnung birgt die Gefahr der Resistenzbildung und führt zu mehr Nebenwirkungen.}

Eine Umstellung von i. v. auf p.o. Gabe sollte erfolgen, wenn der Patient klinisch stabil ist und die orale Nahrungsund Medikamenteneinnahme sichergestellt (orale Sequenztherapie).

Eine Deeskalation ist geboten bei Erregernachweis und zweifelsfreier Diagnose auf eine reduzierte, aber noch wirksame Therapie nach Resistogramm.

Weiteres Vorgehen Bei Therapieversagen sollten eine (erneute) Erregerdiagnostik sowie eine Wiederholung oder Ausweitung der Bildgebung erfolgen. Eine komplette Ausheilung der Pneumonie erfolgt normalerweise binnen 4 Wochen. Bei klinischer Besserung ist ein Kontroll-Röntgen nicht erforderlich. Sollte es erforderlich sein, dann frühestens 2 Wochen nach Erstdiagnose (nicht zu früh kontrollieren!). Bei Risikofaktoren für ein Bronchialkarzinom (insbesondere bei Rauchern $\geq 65$ Jahre) sollte ein Kontroll-Thoraxröntgen vor der Entlassung durchgeführt werden, um eine maligne Raumforderung nicht zu übersehen.

\section{$4 \quad$ Lungenembolie}

Die Lungenembolie ist eine der ernsthaften lebensbedrohlichen Komplikationen bei bettlägerigen Patienten und bei Patienten mit postoperativer Bettlägerigkeit. Die Lungenembolie kann sich in deutlich unterschiedlich starken und differierenden klinischen Symptomen darstellen. Meist handelt es sich um repetitive Ereignisse, bevor es zu einer endgültigen schweren Symptomatik kommt. Die ersten Ereignisse werden meist sowohl vom Patienten als auch vom Klinikpersonal übersehen oder falsch gedeutet. Ursachen sind überwiegend Thrombosen im Bereich der unteren, gelegentlich auch der oberen Extremitäten oder im Bereich des kleinen Beckens, insbesondere nach gynäkologischen oder urologischen Operationen. Auch die üblicherweise durchgeführte postoperative Antikoagulation oder die Antikoagulation bei bettlägerigen Patienten ist nicht immer ausreichend, um sicher eine klinisch relevante Thrombose zu verhindern. In über $90 \%$ der Ereignisse geht der Lungenarterienembolie eine tiefe Beinvenenthrombose voraus, durch eine Einschwemmung der Blutgerinnsel aus peripheren Venen kommt es zu einer teilweisen oder vollständigen Verlegung der Lungenarterien mit daraus resultierender Minderperfusion der Lunge mit Hypoxämie bzw. konsekutiver Rechtsherzbelastung. Todesfälle ereignen sich häufig akut nach Beginn der Symptome, wobei die Schwere der akuten Rechtsherzbelastung den Ausgang entscheidend beeinflusst. Kardiale oder pulmonale Komorbiditäten tragen ebenfalls maßgeblich zur Frühletalität bei Lungenarterienembolie bei. Daher sollte der klinische Verdacht einer Lungenarterienembolie unverzüglich zu einer geeigneten Abklärung führen. Bei hoher klinischer Wahrscheinlichkeit einer Lungenarterienembolie sollte auch bereits vor Diagnosesicherung eine Therapie durch eine geeignete Antikoagulation begonnen werden.

\section{Klinik}

Die Klinik der Lungenembolie besteht in einer plötzlich auftretenden Luftnot, Husten, ggf. Hämoptyse, ansonsten keinerlei Auswurf, kein Fieber, kein Schüttelfrost, jedoch häufig lokalisierten atemabhängigen thorakalen Schmerzen. Dabei zeigt sich eine Tachykardie überwiegend im Sinusrhythmus sowie im weiter fortgeschrittenen Stadium eine Schocksymptomatik mit Blutdruckabfall des systolischen und diastolischen Druckes in Bereiche des positiven Schockindex hinein. Der Patient ist erheblich klinisch kompromittiert mit Angst, Luftnot, Schmerzen und Schweißausbrüchen bis hin zur Eintrübung.

Als Ursachen neben den Thrombosen im Bereich der unteren und oberen Extremitäten haben sich in neuerer Zeit die häufig gelegten zentralvenösen Zugänge im Bereich der $\mathrm{V}$. jugularis und V. subclavia, aber auch der Leiste entwickelt. Hier kann es zusätzlich zu einer infektiösen Thrombose im Bereich der gelegten Fremdkörper kommen. 
Kleinere Lungenembolien verursachen oft nur kurze klinische Symptome, sie sollten als Warnhinweise erkannt werden.

\section{Diagnostik}

Bei klinischem Verdacht auf eine Lungenarterienembolie sollte zunächst eine geeignete Risikostratifizierung erfolgen, bei welcher zwischen hämodynamisch stabilen und instabilen Patienten zu unterscheiden ist. Bei der erstgenannten Gruppe sollte entweder durch den erfahrenen Kliniker oder aber mittels eines geeigneten validierten Scores, z. B. dem Wells-Score, entschieden werden, ob eine weiterführende Diagnostik erforderlich ist (s. Übersicht). Beim hämodynamisch instabilen Patienten sollte umgehend die Verlegung auf eine Überwachungseinheit/Intensivstation sowie eine geeignete Therapie erfolgen.

\section{Validierter klinischer Score zur Ermittlung der klinischen} Wahrscheinlichkeit einer Lungenembolie: Wells-Score (DGA 2015)

- Frühere tiefe Beinvenenthrombose oder Lungenarterienembolie

- Frische Operation oder Immobilisation

- Tumorerkrankung

- Hämoptyse

- Herzfrequenz $>100$ Schläge/min

- Klinische Zeichen einer tiefen Venenthrombose

- Alternative Diagnose unwahrscheinlicher als Lungenarterienembolie

Jedes zutreffende Kriterium wird mit einem Punkt bewertet. Die Punktzahlen werden zusammengezählt, bei $0-1$ Punkten besteht eine nur geringe unter $5 \%$ ige Wahrscheinlichkeit einer Lungenembolie, bei 2-6 Punkten besteht eine mittlere Wahrscheinlichkeit für das Vorliegen einer Lungenembolie, jedoch ist der Cut-off-Punkt bei 4 Punkten für das Bestehen einer Lungenembolie festgelegt. Bei 4 oder weniger Punkten und negativem D-Dimer ist die Wahrscheinlichkeit für das Vorliegen einer Lungenembolie sehr gering. Zwischen 7 und 12 Punkten ist die Wahrscheinlichkeit für das Vorliegen einer Lungenembolie sehr hoch.

Unter Summation der oben genannten Punktewerte ergibt sich bei 1-2 Punkten nur eine geringe Wahrscheinlichkeit einer tiefen Beinvenenthrombose, bei 0 Punkten von unter $5 \%$. Bei mehr als 3 Punkten liegt die Wahrscheinlichkeit einer tiefen Beinvenenthrombose hingegen bei mehr als $80 \%$.

Bei einer nur geringen Wahrscheinlichkeit nach ausgerechnetem Wells-Score und einem negativen Wert für das D-Dimer im Serum ist die Wahrscheinlichkeit des Vorliegens einer tiefen Beinvenenthrombose weitgehend ausgeschlossen.

Hinweise können sich zusätzlich ergeben aus der Durchführung einer Dopplerduplexuntersuchung der tiefen Beinvenen im Bereich des möglicherweise betroffenen Beines. Hier wird überwiegend im angelsächsischen Raum eine sog. 2-Punkt-Methode im Bereich der Leiste und der Kniekehle durchgeführt.

Bei wahrscheinlicher Thrombose im Bereich der tiefen Beinvenen ist dann - bei einer entsprechenden Symptomatik - eine weitere rasche Abklärung notwendig, meist mittels Angio-CT.

D-Dimer-Tests Der D-Dimer-Test ist bezüglich thromboembolischer Ereignisse hochsensitiv, jedoch wenig spezifisch. Daher sollte beim hämodynamisch stabilen Patienten der Test nur bei nicht hoher klinischer Wahrscheinlichkeit durchgeführt werden, da bei niedriger und mittlerer klinischer Wahrscheinlichkeit und normalem D-Dimer-Test keine weitere Diagnostik hinsichtlich einer Beinvenenthrombose oder Lungenarterienembolie mehr erforderlich ist. Im Falle einer hohen klinischen Wahrscheinlichkeit einer Lungenarterienembolie reicht der negative D-Dimer Test jedoch nicht zum definitiven Ausschluss eines thromboembolischen Ereignisses aus, hier ist in jedem Fall eine bildgebende Diagnostik erforderlich.

Pulmonale Bildgebung Das heutzutage überlegene, nahezu überall verfügbare bildgebende Verfahren zur Detektion von Lungenarterienembolien ist die CT-Pulmonalisangiografie (CTPA). Die kombinierte Ventilation-Perfusionsszintigrafie kommt allenfalls noch bei Kontraindikationen für die CTPA bei hämodynamisch stabilen Patienten infrage. Die kombinierte Ventilations-/Perfusionsszintigrafie kann beim hämodynamisch stabilen Patienten als Alternative zur CTPA eingesetzt werden. Bei entsprechender Expertise lässt sich auch mit dem Schall der Lunge eine Lungenarterienembolie diagnostizieren, eine entsprechende Erfahrung weisen jedoch nur wenige Untersucher auf, und es ist zu bezweifeln, dass bei negativem Lungenultraschall bei begründetem Verdacht auf eine Lungenarterienembolie auf eine weitere bildgebende Diagnostik verzichtet werden wird. Eine selektive Pulmonalisangiografie wird kaum noch praktiziert und ist lediglich in Zentren mit erfahrener interventioneller Radiologie verfügbar, bei instabilen Patienten kann die Untersuchung zur lokalen Thrombolyse bzw. Thrombusaspiration oder-fragmentation genutzt werden.

Sonografie der Beinvenen Die Beinvenensonografie gehört beim hämodynamisch stabilen Patienten obligat zur Abklärung des Verdachts einer Lungenarterienembolie. Bei Nachweis einer tiefen Venenthrombose und klinischen Zeichen einer Lungenarterienembolie kann diese bei Patienten mit Kontraindikationen für eine bildgebende Diagnostik (CTPA), 
z. B. bei Schwangerschaft, als bestätigt angesehen werden und eine geeignete Antikoagulation eingeleitet werden.

Echokardiografie Eine Echokardiografie liefert bei klinischem Verdacht einer Lungenarterienembolie und begleitenden Rechtsherzbelastungszeichen einen starken Hinweis für das Vorliegen einer Embolie. Gleichzeitig signalisiert ausgeprägte Rechtsherzbelastung ein hohes Risiko einer frühen Letalität durch die akute Lungenarterienembolie. Zudem können durch die Echokardiografie wichtige kardiale Differenzialdiagnosen wie Vitien, linksventrikuläres Pumpversagen oder Perikardtamponade ausgeschlossen werden. Ein normaler echokardiografischer Befund schließt eine hämodynamisch relevante Lungenembolie mit großer Sicherheit aus, jedoch nicht eine kleinere, hämodynamisch nicht relevante Embolie im Sinne eines prämonitorischen Infarktes.

\section{Therapie \\ Risikostratifizierung}

Bei nachgewiesener Lungenarterienembolie beschreibt die aktuell gültige Leitlinie eine Risikostratifizierung bei hämodynamisch stabilen Patienten. Mittels Anwendung eines validierten klinischen Scores kann zwischen niedrigem, intermediär niedrigem und intermediär hohem Risiko unterschieden werden (PESI, sPESI, s. unten).

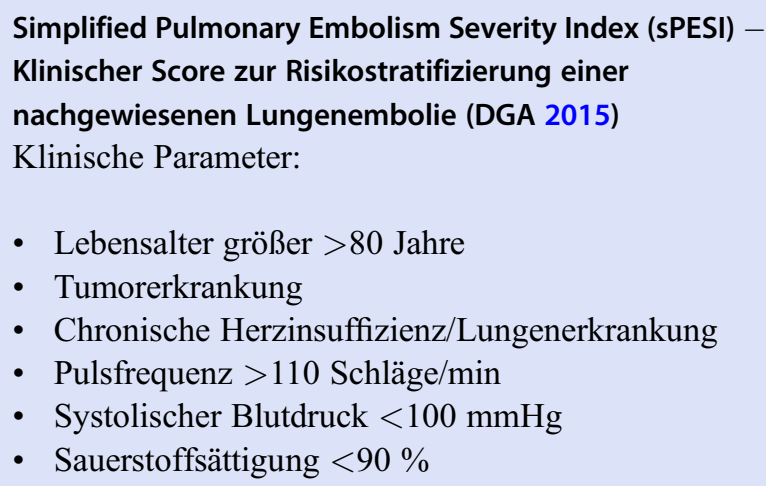

- Lebensalter größer $>80$ Jahre

- Tumorerkrankung

- Chronische Herzinsuffizienz/Lungenerkrankung

- Pulsfrequenz $>110$ Schläge/min

- Systolischer Blutdruck $<100 \mathrm{mmHg}$

- Sauerstoffsättigung $<90 \%$

Jeder Parameter wird mit einem Punkt bewertet, bei 0 Punkten liegt das statistische 30-Tages-Todesrisiko bei 1,0\% (95\% VI: 0,0-2,1\%), bei >1 Punkt liegt das 30-Tages-Todesrisiko schon bedeutend höher bei statistisch 10,9 \% (95\% VI: 8,5-13,2\%).

Die endgültige Risikostratifizierung der Lungenembolie erfolgt dann unter Zusammenschau der Kreislaufsituation, des SPESI, der Echokardiografie sowie kardialer Marker (troponin, proBNP) (DGA 2015).

Ein hohes Risiko liegt demnach vor bei Schock oder Hypotension, sPESI $>1$, einer rechtsventrikulären Dysfunktion in der Echokardiografie sowie der Aktivierung kardialer biochemischer Marker.
Ein intermediäres Risiko liegt vor, wenn außerdem Schock oder Hypotension alle Kriterien der Hochrisikogruppe erfüllt sind.

Ein niedriges Risiko liegt vor bei stabiler Kreislaufsituation ohne Schock oder Hypotension, sPESI von 0, unauffälliger Echokardiografie sowie fehlender Aktivierung von kardialen Markern.

Antikoagulation Bezüglich der Auswahl der Medikamente zur Antikoagulation sowie der Therapiedauer gelten für die Patienten mit Lungenarterienembolie dieselben Empfehlungen wie für die Therapie der tiefen Venenthrombose. Bei Patienten mit niedrigem Risiko kann die Behandlung der Lungenembolie ambulant erfolgen. Die Antikoagulation sollte bei begründetem klinischen Verdacht einer Lungenarterienembolie, spätestens aber nach Diagnosestellung derselben begonnen werden. Auch der Nachweis einer tiefen Venenthrombose erfordert den sofortigen Beginn einer Antikoagulation unter Berücksichtigung der Komorbiditäten und Kontraindikationen. Eine Immobilisation von Patienten mit einer tiefen Venenthrombose es nicht mehr üblich und erforderlich. Zur Vermeidung eines postthrombotischen Syndroms sollte jedoch vor Mobilisation mit einer Kompressionstherapie durch Wickelung des Beines bzw. Anpassung eines geeigneten Kompressionsstrumpfes begonnen werden. Durch die Antikoagulation sollen eine Lungenarterienembolie bzw. wiederholte Ereignisse verhindert werden. Des Weiteren kommt es bei ausbleibender Antikoagulation zu einem appositionellen Thrombuswachstum, welches durch die Blutverdünnung verhindert werden soll. Durch eine Aktivierung der körpereigenen Fibrinolyse soll langfristig eine Auflösung der Embolie bzw. des Thrombus mit Verringerung des postthrombotischen Syndroms erreicht werden.

Die initiale Antikoagulation erfolgt in aller Regel mit einem parenteralen Antikoagulans (NMH oder Fondaparinux). Im Verlauf kann dann auf eine orale Antikoagulation mittels Vitamin-K-Antagonist oder direkten orale Antikoagulanzien (DOAKs) umgestellt werden. Zu Beginn der Antikoagulation ist ein Basisgerinnungsstatus sowie die Bestimmung von Thrombozytenzahl und Nierenfunktion obligat erforderlich. Bei Therapie mit einem unfraktionierten Heparin sollte im Verlauf die Thrombozytenzahl zur rechtzeitigen Detektion einer möglicherweise bestehenden heparininduzierten Thrombozytopenie (HIT II) erfolgen. Bei akuter HIT sind die in dieser Situation zugelassenen Antikoagulanzien einzusetzen.

Die in Deutschland zur Therapie der Venenthrombose und der Lungenembolie zugelassenen Medikamente sind in Tab. 5 aufgeführt.

Der hämodynamisch instabile Patient mit Lungenarterienembolie sollte mittels Gabe von unfraktioniertem Heparin (UFH) als Bolus, gefolgt von einer PTT-gesteuerten Gabe behandelt werden, da hier im Verlauf die Möglichkeit einer 
Tab. 5 Initiale Antikoagulation und Erhaltungstherapie bei tiefer Venenthrombose/Lungenembolie. (Mod. nach DGA 2015)

\begin{tabular}{|c|c|c|c|c|}
\hline Wirkstoff & Präparat & Initiale Dosis/Startmedikation & Erhaltungsdosis $^{\mathrm{a}}$ & Anwendung \\
\hline \multicolumn{5}{|c|}{ Niedermolekulare Heparine (NMH) } \\
\hline Certoparin & $\begin{array}{l}\text { Mono-Embolex } \\
8000 \text { I.E. Therapie }\end{array}$ & $8000 \mathrm{IE}$ & $8000 \mathrm{IE}$ & 2-mal/Tag s.c. \\
\hline \multirow[t]{2}{*}{ Dalteparin } & Fragmin & $100 \mathrm{IE} / \mathrm{kg} \mathrm{KG}$ & $100 \mathrm{IE} / \mathrm{kg} \mathrm{KG}$ & 2-mal/Tag s.c. \\
\hline & Fragmin & $200 \mathrm{IE} / \mathrm{kg} \mathrm{KG}$ & $200 \mathrm{IE} / \mathrm{kg} \mathrm{KG}$ & 1-mal/Tag s.c. \\
\hline Enoxaparin $^{\mathrm{b}}$ & Clexane & $1,0 \mathrm{mg} / \mathrm{kg} \mathrm{KG}$ & $1,0 \mathrm{mg} / \mathrm{kg} \mathrm{KG}$ & 2-mal/Tag s.c. \\
\hline \multirow[t]{2}{*}{ Nadroparin } & Fraxiparine & $0,1 \mathrm{ml} / 10 \mathrm{~kg} \mathrm{KG}$ & $0,1 \mathrm{ml} / 10 \mathrm{~kg} \mathrm{KG}$ & 2-mal/Tag s.c. \\
\hline & Fraxodi & $0,1 \mathrm{ml} / 10 \mathrm{~kg} \mathrm{KG}$ & $0,1 \mathrm{ml} / 10 \mathrm{~kg} \mathrm{KG}$ & 1-mal/Tag s.c. \\
\hline \multirow[t]{2}{*}{ Reviparin } & Clivarin & $0,6 \mathrm{ml}$ bei $\mathrm{KG} 45-60 \mathrm{~kg}$ & $\begin{array}{l}0,6 \mathrm{ml} \text { bei } \mathrm{KG} 45- \\
60 \mathrm{~kg}\end{array}$ & 2-mal/Tag s.c. \\
\hline & Clivarin & $0,6 \mathrm{ml}$ bei $\mathrm{KG}>60 \mathrm{~kg}$ & $0,6 \mathrm{ml}$ bei $\mathrm{KG}>60 \mathrm{~kg}$ & 1-mal/Tag s.c. \\
\hline Tinzaparin & innohep & $175 \mathrm{IE} / \mathrm{kg} \mathrm{KG}$ & $175 \mathrm{IE} / \mathrm{kg} \mathrm{KG}$ & 1-mal/Tag s.c. \\
\hline \multicolumn{5}{|l|}{ Pentasaccharid } \\
\hline Fondaparinux & Arixtra & $\begin{array}{l}7,5 \mathrm{mg} 5 \mathrm{mg} \text { bei } \mathrm{KG}<50 \mathrm{~kg} \\
10 \mathrm{mg} \text { bei } \mathrm{KG}>100 \mathrm{~kg}\end{array}$ & $\begin{array}{l}7,5 \mathrm{mg} 5 \mathrm{mg} \text { bei } \mathrm{KG}< \\
50 \mathrm{~kg} \\
10 \mathrm{mg} \text { bei } \mathrm{KG}> \\
100 \mathrm{~kg}\end{array}$ & 1-mal/Tag s.c. \\
\hline \multicolumn{5}{|c|}{ Unfraktionierte Heparine (UFH) } \\
\hline Heparin-Calcium & Heparin-Calcium & Bolus $5000 \mathrm{IE}$, dann 15-20 IE/kg KG/h & ca. $15-20 \mathrm{IE} / \mathrm{kg} \mathrm{KG} / \mathrm{h}$ & $\begin{array}{l}\text { Bolus i. v., dann i. v. } \\
\text { Infusion oder 2-mal/Tag s. } \\
\text { c. }\end{array}$ \\
\hline \multicolumn{5}{|c|}{ Direkte orale Antikoagulanzien } \\
\hline Dabigatranetexilat & Pradaxa & NMH, UFH oder FDX mind. 5 Tage & $150 \mathrm{mg}$ & 2-mal/Tag oral \\
\hline Rivaroxaban & Xarelto & $15 \mathrm{mg}$ 2-mal/Tag oral 3 Wochen & $20 \mathrm{mg}$ & 1-mal/Tag oral \\
\hline Apixaban & Eliquis & $10 \mathrm{mg}$ 2-mal/Tag oral 1 Woche & $\begin{array}{l}5 \mathrm{mg} \text { (nach } 6 \text { Monaten } \\
2,5 \mathrm{mg} \text { ) }\end{array}$ & 2-mal/Tag oral \\
\hline Edoxaban & Lixiana & NMH, UFH oder FDX mind. 5 Tage & $60 \mathrm{mg}$ & 1-mal/Tag oral \\
\hline \multicolumn{5}{|c|}{ Vitamin-K-Antagonisten ${ }^{c}$} \\
\hline Phenprocoumon & $\begin{array}{l}\text { Marcumar } \\
\text { Falithrom }\end{array}$ & $\begin{array}{l}6 \mathrm{mg} / \text { Tag am } 1 . \text { und } 2 . \text { Tag; NMH, UFH } \\
\text { oder FDX parallel bis INR } \geq 2,0\end{array}$ & $\begin{array}{l}\text { ca. } 1,5-4,5 \mathrm{mg} \text { allein } \\
\text { weiter bei INR } \geq 2,0\end{array}$ & Oral, nach INR \\
\hline Warfarin-Natrium & Coumadin $5 \mathrm{mg}$ & $\begin{array}{l}2,5-5 \mathrm{mg} / \mathrm{Tag} \text { am } 1 . \text { und } 2 . \text { Tag; NMH, } \\
\text { UFH oder FDX parallel bis INR } \geq 2,0\end{array}$ & $\begin{array}{l}\text { ca. } 2,5-10,0 \mathrm{mg} \text { allein } \\
\text { weiter bei INR } \geq 2,0\end{array}$ & Oral, nach INR \\
\hline
\end{tabular}

medikamentösen Thrombolyse offengehalten wird (Konstantinidis et al. 2014).

Reperfusionstherapie Die Entscheidung für eine Reperfusionstherapie im Sinne einer medikamentösen Thrombolyse hängt von der hämodynamischen Situation des Patienten ab. Der hämodynamisch instabile Patient mit Lungenarterienembolie sollte eine sofortige Reperfusionstherapie erhalten (Tab. 6). Bei hämodynamisch stabilen Patienten ist die medikamentöse Thrombolyse lediglich notwendig bei im Verlauf eintretender hämodynamischer Instabilität (Konstantinidis et al. 2014).
Tab. 6 Zugelassene Substanzen und Dosierungen zur Therapie der akuten Lungenembolie (DGA 2015)

\begin{tabular}{l|l}
$\begin{array}{l}\text { Alteplase } \\
\text { (rt-PA) }\end{array}$ & $\begin{array}{l}\text { Bolus-Injektion von } 10 \mathrm{mg} \text { über } 1-2 \mathrm{~min} \text {, gefolgt von } \\
90 \mathrm{mg} \text { über } 2 \mathrm{~h} \text { oder } 100 \mathrm{mg} \text { über } 2 \mathrm{~h} \text { oder akzeleriert: } \\
0,6 \mathrm{mg} / \mathrm{kg} \text { über } 15 \mathrm{~min}\end{array}$ \\
\hline Streptokinase & $\begin{array}{l}250.000 \mathrm{IE} \text { über } 30 \mathrm{~min}, \text { gefolgt von } 100.000 \mathrm{IE} / \mathrm{h} \\
\text { über } 12-24 \mathrm{~h} \text { oder akzeleriert: } 1,5 \mathrm{Mio} \text {. IE über } 2 \mathrm{~h}\end{array}$ \\
\hline Urokinase & $\begin{array}{l}4400 \mathrm{IE} / \mathrm{kg} \mathrm{KG} \text { über } 10 \mathrm{~min}, \text { gefolgt von } 4400 \mathrm{IE} / \mathrm{kg} / \\
\mathrm{h} \text { über } 12-24 \mathrm{~h} \text { oder akzeleriert: } 3 \mathrm{Mio} \text {. IE über } 2 \mathrm{~h}\end{array}$
\end{tabular}

\section{Schlafapnoe-Syndrom}

Das Schlafapnoe-Syndrom hat seit Ende der 1980er-Jahre klinisches, diagnostisches und therapeutisches Interesse gefunden, insbesondere unter zwei Gesichtspunkten, nämlich der Entwicklung von Folgeerkrankungen wie Myokard- 
infarkt oder zerebralem Insult sowie der Entwicklung typischer Klinik wie Tagesmüdigkeit, imperativem Einschlafdrang mit Sekundenschlaf, Konzentrationsstörungen, Merkfähigkeitsstörungen, Aggressivität, Depression und Entwicklung entsprechender sozialer Kompensationsmechanismen.

\section{Klinik}

Die Erkrankung ist klinisch mehr oder minder manifest bei etwa 4-5\% der männlichen Bevölkerung über 40 Jahren, bei Frauen beginnt die Klinik etwas später nach Einsetzen der Menopause. Risikofaktoren sind Adipositas, Mikrognathie im Bereich des Unterkiefers, Tonsillenhypertrophie beidseits, eingeschränkte bis aufgehobene Nasenatmungsmöglichkeit.

Es sollte jedoch nicht vergessen werden, dass auch bei Kindern und Jugendlichen überwiegend über die Tonsillenhypertrophie eine entsprechende Symptomatik des Schnarchens und der obstruktiven Apnoen auftreten kann. Dies kann zu entsprechenden Gedeihstörungen der Kinder und Jugendlichen führen, mit deutlichen Leistungseinbußen auch im Schulalltag.

Folgen sind ausgeprägte Tagesmüdigkeit mit Konzentrations- und Merkfähigkeitsstörungen. Laborchemisch zeigt sich bei deutlichen Sauerstoffsättigungsabfällen in der Nacht eine Polyglobulie. Des Weiteren zeigen sich leichte Zeichen einer pulmonalen Hypertonie, die Entwicklung einer arteriellen Hypertonie mit fehlender Nachtabsenkung, Herzrhythmusstörungen überwiegend mit AV-Blockierungen. Zusätzlich besteht meistens ein lautes sozial störendes Schnarchen. Die Entwicklung ergibt dann im weiteren Verlauf eine zunehmende Häufigkeit von Atempausen über 10 Sekunden. Bei Atempausenhäufigkeiten über 20 Atempausen/h entsteht meist eine Therapiebedürftigkeit durch sich entwickelnde Tagesklinik. Abzugrenzen von der Schlafapnoe und dem fortgeschrittenem Schnarchen ist die alveoläre Hypoventilation bei Obesitas (Pickwick-Syndrom). Hierbei kommt es durch übermäßige Adipositas und Zwerchfellhochstand zunächst während des Schlafes, später auch am Tage zu einer nicht ausreichenden Ventilation mit $\mathrm{PaCO}_{2}$-Anstieg bis in die $\mathrm{CO}_{2}$-Narkose.

\section{Diagnostik}

Die Diagnose ergibt sich zunächst weitgehend aus der typischen Klinik und der Fremdanamnese des Bettpartners. In der Polygrafie und Polysomnografie erfolgt dann die weitere Einteilung in die verschiedenen Schweregrade der Diagnosen Schnarchen, Upper-airway-resistance-Syndrom, alveoläre Hypoventilation und obstruktives Schlafapnoe-Syndrom.

\section{Therapie}

Die Therapieentscheidung zu einer nasalen nächtlichen Überdruckatmung (nCPAP) erfolgt entweder aufgrund der erheblichen Einschränkung im privaten oder beruflichen Leben durch die Tagesmüdigkeit und Einschlafneigung oder durch das Bestehen zusätzlicher kardiovaskulärer Risikofaktoren, wie koronare Herzkrankheit (KHK), Hypertonie, Diabetes mellitus und abgelaufenen zerebralen Insulten. $\mathrm{Ob}$ bei einer Herzinsuffizienz bei Bestehen von zentralen und peripheren Obstruktionen eine Verbesserung herbeigeführt werden kann, ist nach der augenblicklichen Studienlage noch nicht endgültig geklärt.

Ursache der obstruktiven Schlafapnoe ist eine Einengung im Bereich des Pharynxeingangs durch anatomische Veränderungen, aber auch durch physiologische neurologische Störungen der Innervation der Pharynxmuskulatur. Hinzu kommen anatomische Veränderungen des weichen Gaumensegels, der Ovula, des Kehldeckels und auch des Nasenflows durch Einengung der inneren Nasenöffnungen. Neben der nasalen nächtlichen Überdruckatmung durch das nCPAP-Gerät, welches mindestens 4-6 h nächtlich getragen werden sollte, ist auch an operative Maßnahmen zu denken. Eine Verbesserung der klinischen Situation der Tagesmüdigkeit gelingt trotz suffizienter Therapie nicht immer (DGSM 2017).

\section{$6 \quad$ Respiratorische Insuffizienz als Manifestation einer neurologischen Erkrankung}

Durch die medizintechnische Entwicklung von kleinen, leisen, wartungsarmen Beatmungsgeräten ist es möglich geworden, Patienten mit ausgeprägter Störung der Atempumpe bei neuromuskulären Erkrankungen ein langfristiges Überleben zu sichern. Die neuesten auf dem Markt befindlichen Beatmungsgeräte sind in der Lage, weitgehend alle relevanten Beatmungsformen durchzuführen, um dem Patienten eine angenehme Beatmungssituation mit Normalisierung sowohl des Gefühls von Luftnot als auch der Blutgase zu ermöglichen.

Indikation für die Einleitung einer Therapie sind die zunehmenden Luftnotgefühle eines Patienten als Zeichen der ansteigenden $\mathrm{PaCO}_{2}$-Werte mit respiratorischer Azidose mit der Notwendigkeit einer Hyperventilation und vermehrten Atemarbeit. Dies gelingt dem Patienten aber nicht aufgrund der gestörten Atempumpe durch die neuromuskuläre Erkrankung. Der Patient entwickelt eine zunehmende Eintrübung und Kopfschmerzsymptome. Hier sollte dann in Absprache mit einem entsprechend erfahrenen Zentrum unter Einbeziehung des Patienten, der Angehörigen und der mitbetreuenden Pflegekräfte ein Konzept zur Beatmung entwickelt werden. Zunächst ist an eine nächtliche nichtinvasive Beatmung durch eine Nase-Mund-Maske und ein entsprechendes Beatmungsgerät zu denken. Hierbei ist zu beachten, dass der Patient die Nasenmaske selbst aufgrund der neuromuskulären Störung meist nicht mehr korrekt aufsetzen kann. Hier sind 
entsprechende Hilfestellungen regelmäßig notwendig. Im weiteren Verlauf bei Verschlechterung der Symptomatik ist auch eine Benutzung der nichtinvasiven Beatmung tagsüber zunehmend erforderlich. Die Schwierigkeiten der nichtinvasiven Beatmung bestehen darin, dass in dieser Zeit Sprechen und Nahrungs- und Flüssigkeitsaufnahme nicht möglich ist. Zusätzlich kommt es bei manchen Patienten bei notwendigen hohen Beatmungsdrücken und Insuffizienz der Ösophagussphinktermuskulatur zu einer Anfüllung des Magen-DarmTraktes mit Luft durch die Beatmung mit entsprechenden Beschwerden.

Im weiteren Verlauf sollte dann frühzeitig mit dem Patienten und dem häuslichen Behandlungsteam besprochen werden, dass die Umstellung der nichtinvasiven auf eine invasive Beatmung über ein Tracheostoma notwendig ist. Unter dieser Therapie (bei noch fehlenden Schluckstörungen) kann der Patient noch essen und trinken und auch mit entsprechender Kanüle sprechen.

Der Pflegeaufwand eines solchen Systems ist erheblich und bei vollständiger Abhängigkeit des Patienten von einer Beatmungsmaschine über $24 \mathrm{~h}$ am Tag ist auch der personelle Aufwand einer 24-h-Betreuung in häuslicher Umgebung zu beachten. Die Durchführung einer solchen Therapie beinhaltet einen erheblichen organisatorischen und materiellen Aufwand und kann und sollte nur durch entsprechende Einrichtungen eingeleitet und auch durchgeführt werden.

Typische Erkrankungen, die zu einer solchen Therapieform führen können, sind die amyotrophe Lateralsklerose (ALS), die Poliomyelitis, Polyradikulitiden, myasthene Syndrome, Muskeldystrophien, sowie Myopathien und Myositiden aller Art.

Die Therapie der chronischen respiratorischen Insuffizienz im Rahmen neuromuskulärer Erkrankungen umfasst die regelmäßige Dokumentation von Vitalkapazität, Atemproblemen (mittels ALSFRS - ALS Functional Rating Scale) und im Verlauf regelmäßigen BGA-Kontrollen. Supportiv sollten Atemgymnastik, Klopfmassagen, schleimlösende Maßnahmen durch ausreichende Flüssigkeitszufuhr und ggf. die Gabe von Acetylcystein erfolgen. Bei unzureichendem Hustenstoß, welcher regelmäßig mittels Peak Cough Flow bestimmt werden sollte, kann eine Hustenhilfe (mechanischer Insufflator/ Exsufflator, „,cough assist“) zur Mobilisation von Sekreten angepasst werden. Bei entsprechendem Patientenwunsch kann auch im Verlauf eine Tracheotomie zur Vereinfachung der Sekretabsaugung diskutiert werden.

Im Falle einer zunehmenden Symptomatik mit respiratorischer Erschöpfung unter stationären Bedingungen ist bei Nachweis alveolärer Hypoventilationen bzw. einer Hyperkapnie die Anpassung einer zunächst nicht invasiven Beatmung erforderlich sowie bei entsprechendem Patientenwunsch bei vollständiger Erschöpfung auch die invasive Beatmung nach Tracheotomie und Anlage eines endständigen Tracheostomas. Die genannten Maßnahmen führen alle- samt zu einer Verbesserung der Lebensqualität und sekundär auch, insbesondere bei Einleitung einer invasiven Beatmung über ein Tracheostoma, zu einer Verlängerung der Überlebensdauer. Frühzeitig sollte besprochen und dokumentiert werden, ob eine notfallmäßige Intubation gewünscht ist!

\section{$7 \quad$ Erkrankungen mit gleichzeitiger pulmonaler und neurologischer Manifestation}

Hier sind v. a. systemische Vaskulitiden wie die (eosinophile) Granulomatose mit Polyangiitis und die Sarkoidose zu nennen. Dabei ist eine intensive Zusammenarbeit insbesondere aus therapeutischer Sicht zwischen Pneumologen und Neurologen notwendig, damit der jeweils klinisch führende Anteil der Erkrankung entsprechend den Richtlinien behandelt werden kann.

Die Diagnostik bei pulmonalen Manifestationen erfolgt aufgrund der leichteren Zugänglichkeit zu histologischen Proben am ehesten durch Bronchoskopie mit bronchoalveolärer Lavage, transbronchialer Biopsie oder auch durch Mediastinoskopie. Transthorakale CT-gesteuerte Punktionen sind ebenfalls manchmal richtungsweisend (Fraser et al. 1994).

\section{Neurologische Symptomatik einer primär pulmonalen Erkrankung}

Eine neurologische Symptomatik muss nicht immer durch eine neurologische Diagnose ausgelöst werden. Hierzu zählen z. B. die bereits oben genannten Veränderungen wie Sekundenschlaf, Konzentrations- und Merkfähigkeitsstörungen und Depression durch fortgeschrittenes Schnarchen mit Upper-airway-resistance-Syndrom, dem obstruktiven Schlafapnoe-Syndrom und der alveolären Hypoventilation bei Obesitas (Pickwick-Syndrom).

Eine Myasthenia gravis tritt häufig in Assoziation mit Thymomen, auch malignen Thymomen im vorderen Mediastinalbereich auf.

Das Lambert-Eaton-Syndrom ist bei kleinzelligen Bronchialkarzinomen eine nicht häufige, aber typische Paraneoplasie. Die Liste paraneoplastisch-neurologischer Symptomenkomplexe beim kleinzelligen Bronchialkarzinom ist lang. Daneben besteht auch die Gefahr der diffusen zerebralen oder meningealen Metastasierung.

Eine neurologische bzw. psychotische Symptomatik durch eine Hyperkalzämie ist nicht selten, entweder verursacht durch ein ossär metastasierendes Bronchialkarzinom oder auch durch einen paraneoplastischen Pseudohyperparathyreoidismus. 
Im weiteren Verlauf schwerer bronchopulmonaler Erkrankungen kann es auch durch die sich entwickelnde Hypoxämie und nicht nur durch die relativ frühzeitig entstehende Hyperkapnie zu erheblicher neurologischer Symptomatik bis zur Bewusstlosigkeit kommen. Hier sind die klinische Untersuchung, die Blutgasanalyse, typische Laborbefunde und das Röntgenbild überwiegend richtungweisend.

\section{ZNS-Beteiligung beim Lungenkarzinom}

Das Lungenkarzinom ist bei Frauen der dritt-, bei Männern der zweithäufigste maligne Tumor in den deutschsprachigen Ländern. Das mediane Erkrankungsalter liegt zwischen 68 und 70 Jahren. Hauptrisikofaktor ist Rauchen.

Ein routinemäßiges Screening asymptomatischer Risikopersonen mittels einer Computertomografie kann Lungenkarzinome in frühen Stadien erkennen und die Mortalität bei Rauchern und in noch größerem Ausmaß bei Raucherinnen senken, ist aber in den deutschsprachigen Ländern bisher nicht als Früherkennungsprogramm implementiert.

Die Therapie des nichtkleinzelligen Lungenkarzinoms hat in den letzten Jahren erhebliche Fortschritte durch die Entwicklung der sog. Tyrosinkinase-Inhibitoren bzw. Einführung der Immuntherapie gemacht. Noch bis vor Kurzem in zwei wesentlichen Diagnosen (kleinzelliges und nichtkleinzelliges Lungenkarzinom) zusammengefasst, wird das Lungenkarzinom heute in mindestens zwei Dutzend biologisch unterschiedliche Entitäten mit individualisierten Behandlungskonzepten eingeteilt. Die Prognose der Patienten wird vom Stadium, dem Genotyp, der Histologie, dem Geschlecht, dem Allgemeinzustand und der Komorbidität bestimmt. Tumorgewebe wird routinemäßig mittels molekularpathologischer Untersuchungen nach sog. Treibermutationen untersucht. Therapieoptionen v. a. des nichtkleinzelligen Lungenkarzinoms sind Operation, Bestrahlung und systemische Therapie, häufig kombiniert als multimodales Konzept. Patienten mit nichtkleinzelligem Lungenkarzinom haben in frühen und in einem Teil der lokal fortgeschrittenen Stadien einen kurativen Therapieanspruch. Für die große Mehrzahl von Patienten im Stadium IIIB/IV ist die Therapie nicht kurativ. In den letzten Jahren hat die Integration von Immuncheckpoint- und Kinase-Inhibitoren im Zusammenhang mit prädiktiven Biomarkern die Prognose vieler Patienten deutlich verbessert. Weiterhin stehen Zytostatika, AngiogeneseInhibitoren, lokale Therapien und unterstützende Maßnahmen zur Verfügung (Griesinger et al. 2017).

Kleinzellige Lungenkarzinome (SCLC - „small cell lung cancer") machen etwa $12-15 \%$ der Lungenkarzinome aus. In Deutschland erkranken jährlich ca. 7000-8000 Personen. Die Erkrankung ist charakterisiert durch eine hohe Zellteilungsrate und rasche Wachstumsprogredienz. Diese biologischen
Tab. 7 Häufigkeit von Hirnmetastasen im Vergleich zum histologischen Subtyp des Bonchialkarzinoms

\begin{tabular}{|l|l|}
\hline Bronchialkarzinomsubtyp & Häufigkeit von Hirnmetastasen (\%) \\
\hline Plattenepithelkarzinom & 13,7 \\
\hline Kleinzelliges Karzinom & 30,5 \\
\hline Adenokarzinom & 25,4 \\
\hline Großzelliges Karzinom & 29,4
\end{tabular}

Attribute begründen die hohe Sensibilität des Tumors gegenüber Chemo- und Strahlentherapie. Andererseits führen sie auch zu einer frühzeitigen Disseminierung und hohen Rezidivraten. In den Stadien I-III (,very limited disease“, „limited disease") besteht ein kurativer Therapieanspruch. Die Therapie ist multimodal mit Einbeziehung von Operation, medikamentöser Tumortherapie und Bestrahlung (Wolf et al. 2017).

Bronchialkarzinome zeigen sich aufgrund der häufig nur geringen lokalen Beschwerdesymptomatik im Bronchialsystem und der Lunge häufig durch Hirnmetastasen.

Tab. 7 zeigt die Häufigkeit von Hirnmetastasen im Vergleich zum histologischen Subtyp des Bronchialkarzinoms. Dabei ist zu beachten, dass viele Bronchialkarzinome nicht nur einer einheitlichen Histologie gehorchen.

Das mediane Überleben nach Diagnosestellung beträgt einen Monat ohne Therapie und 2 Monate bei symptomatischer Behandlung mit Steroiden. Die Diagnostik der Hirnmetastasen sollte neben einer eingehenden klinisch-neurologischen Untersuchung mit besonderem Augenmerk auf Zeichen erhöhten intrakraniellen Drucks ein MRT des Gehirns ohne/mit gadoliniumhaltigem Kontrastmittel umfassen.

Im Falle symptomatischer Hirnmetastasen oder eines erkennbaren fokalen Ödems sollte eine antiödematöse Therapie mit Dexamethason eingeleitet werden. Die wesentlichen Therapiemodalitäten umfassen Resektion, Radiochirurgie, fraktionierte Strahlentherapie, z. B. als stereotaktisch geführte, lokale Strahlentherapie oder als Ganzhirnbestrahlung, und medikamentöse Tumortherapie (DGN 2019). Bei der Beurteilung des Effekts lokaler Therapien in klinischen Studien ist zu beachten, dass die mediane Überlebenszeit nicht nur durch die Wirksamkeit der Behandlung der Hirnmetastasen beeinflusst wird. Mehr als die Hälfte der Patienten verstirbt nicht an den Hirnmetastasen, sondern an den Folgen der systemischen Tumorprogression (Soffietti et al. 2006). Bei Patienten mit ungünstigen prognostischen Faktoren und entsprechend kurzer Lebenserwartung ist es gerechtfertigt, die Therapie auf supportive Maßnahmen zu beschränken. 


\section{Facharztfragen}

1. Gibt es wesentliche Unterschiede in der medikamentösen Therapie von Asthma und chronisch obstruktiver Bronchitis?

2. Welches bildgebende Verfahren sollte bei der Diagnostik von Hirnmetastasen verwendet werden?

3. Was sind die Leitsymptome des obstruktiven SchlafApnoe-Syndroms?

4. Nennen Sie Möglichkeiten der Beatmung bei neuromuskulären Erkrankungen.

5. Welchem Hauptwirkungsprinzip bedient sich die inhalative Therapie bei Asthma bronchiale?

6. Nennen Sie drei wichtige Allgemeinmaßnahmen zur Therapie der COPD (explizit keine medikamentösen Therapiemaßnahmen).

7. Nennen Sie zwei Antibiotika, die sich zur Behandlung atypischer Pneumonieerreger eignen.

\section{Literatur}

Bauer TT, Ewig S, Marre R, Suttorp N, Welte T (2006) CRB-65 predicts death from community-acquired pneumonia. CAPNETZ Study Group J Intern Med 260(1):93-101

Bodmann et al (2018) S2k-Leitlinie Kalkulierte parenterale Initialtherapie bakterieller Erkrankungen bei Erwachsenen - Update 2018. Paul-Ehrlich-Gesellschaft für Chemotherapie (PEG). Zugegriffen am 20.12.2019

Buhl et al (2017) S2k-Leitlinie zur Diagnostik und Therapie von Patienten mit Asthma . Deutsche Gesellschaft für Pneumologie und Beatmungsmedizin, Deutsche Atemwegsliga. Stand September 2017. Zugegriffen am 21.11.2017

Deutsche Gesellschaft für Neurologie e.V. (DGN), Hirnmetastasen und Meningeosis neoplastic Registrierungsnummer: 030-060, Entwicklungsstufe: S2k. Zugegriffen am 20.12.2019

DGA (Deutsche Gesellschaft für Angiologie-Gesellschaft für Gefässmedizin) (2015) AWMF Leitlinien-Register Nr. 065/002 Klasse S2k Diagnostik und Therapie der Venenthrombose und der Lungenembolie, 2015. Zugegriffen am 01.01.2020
DGP, DGI, PEG (2015) S3-Leitlinie Pneumonie, ambulant erworben, Behandlung und Prävention von erwachsenen Patienten. Deutsche Gesellschaft für Pneumologie und Beatmungsmedizin (DGP), Deutsche Gesellschaft für Infektiologie (DGI), Paul-Ehrlich-Gesellschaft für Chemotherapie (PEG). Stand Dezember 2015. Zugegriffen am 20.12.2019

DGSM (Deutsche Gesellschaft für Schlafforschung und Schlafmedizin) (2017) S3-Leitlinie: Nicht erholsamer Schlaf - Schlafstörungen. http://awmf.org. Zugegriffen am 01.01.2020

Fraser R, Pare JA et al (1994) Synopsis of diseases of the chest, 2nd edn. Saunders, Philadelphia, S $640 \mathrm{ff}, 733 \mathrm{ff}$

Global Initiative for Asthma (GINA) (2017) Global Strategy for Asthma Management and Prevention (2017 update). Global Initiative for Asthma (GINA). Stand Januar 2017. Zugegriffen am 31.01.2018

Global Initiative for Asthma (GINA) (2019) Global Strategy for Asthma Management and Prevention (2019 update). Stand Januar 2019. Zugegriffen am 20.12.2019

Griesinger et al (2017) Leitlinie Lungenkarzinom, nicht-kleinzellig (NSCLC). Deutsche Gesellschaft für Hämatologie und Medizinische Onkologie (DGHO). Stand April 2017. Zugegriffen am 20.12.2019

Konstantinidis S et al (2014) ESC guidelines on the diagnosis and management of acute pulmonary embolism. Eur Heart J 35:30333069k

Matthys, Seeger (2008) Klinische Pneumologie. Springer, ISBN 978-3540-37682-8

NVL - AWMF (2018) Google 2018. 10.6101/AZQ/000400. Zugegriffen am 20.12.2019

Soffietti et al (2006) EFNS Guidelines on disgnosis and treatment of brain metastases: report of an EFNS Task Force. First published: 23 June 2006. https://doi.org/10.1111/j.1468-1331.2006.01506

Vogelmeier et al (2018a) S2k-Leitlinie zur Diagnostik und Therapie von Patienten mit chronisch obstruktiver Bronchitis und Lungenemphysem (COPD). Deutsche Gesellschaft für Pneumologie und Beatmungsmedizin (DGP). Stand Januar 2018. Zugegriffen am 20.12.2019

Vogelmeier et al (2018b) Global Strategy for the Diagnosis, Management, and Prevention of Chronic Obstructive Lung Disease 2018 Report. Global Initiative for Chronic Obstructive Lung Disease (GOLD). Stand Januar 2018. Zugegriffen am 20.12.2019

WHO (2018) The top 10 causes of death. WHO. Stand Mai 2018. Zugegriffen am 20.12.2019

Wolf et al(2017) Leitlinie Lungenkarzinom, kleinzellig (SCLC). Deutsche Gesellschaft für Hämatologie und Medizinische Onkologie (DGHO). Stand April 2017. Zugegriffen am 20.12.2019 